\title{
Moving Object Detection Based on Adaptive Background Updating Algorithm
}

\author{
ZhiyongTang $^{\mathrm{a}}$, Zhenji Yang ${ }^{\mathrm{b}}$, Kun $\mathrm{Liu}^{\mathrm{c}}$ andZhongcaiPei ${ }^{\mathrm{d}}$ \\ Automation Science \& Electrical Engineering, Beihang University, Beijing, 100191, China. \\ a 57523523@qq.com, bryanyzj1717@163.com, ${ }^{c} 356900463 @ q q . c o m,{ }^{d} 64850423$ @qq.com
}

Keywords: Background Updating; Background Subtraction; Moving Object Detection;

Abstract. Background modeling and subtraction method is an important part in motion detection. In this paper, we propose a novel moving object detection method based on four frame difference arithmetic of adaptive background updating algorithmto build the background model. The method employs dynamic threshold in order to adapt to the sudden changes in lights. According to different labels in background, this paper defines different updating rate, and analyzes the image features of moving object area. Experimental results show that, the algorithm can effectively remove noise and rapidly respond to practical scene changes.

\section{Introduction}

In many image processing and computer vision in the scene, an important preprocessing step is to segment moving foreground objects from the static background.Detection of moving objects in video sequence analysis is always very important, with the purpose of the exercise area is extracted from the video sequence [1]. It is an important for object classification, motion analysis, tracking and post-processing image understanding.However, since the complex dynamic scenes, such as weather and lighting, moving object detection in video sequences become a challenging task.

Many methods have been proposed in the literaturesto deal with such multi-channel scenes [2-5]. Popular idea is in time to sample distribution, multi-channel model parameters or nonparametric method, so learn to the changes of background model can tolerate background scenes.Other major challenges in background subtraction are illuminationchange, camera shake, intermittent object motion and shadows. The key point of this method is tofind out an efficient and practical background updating algorithm.

\section{Proposed Algorithm}

\subsection{Traditional background subtraction method}

Background subtraction including background extraction and update method, background subtraction, motion detection [6], binarization [7], morphological changes, and other components, which is shown in Fig. 1. First, the current frame by subtracting the background image and a background model acquired, and in the process which will be regularly updated background image. Finally, perform the necessary post-processing of the template, which means that the small morphological filtering to remove noise area template, then smooth and part of the image boundary.

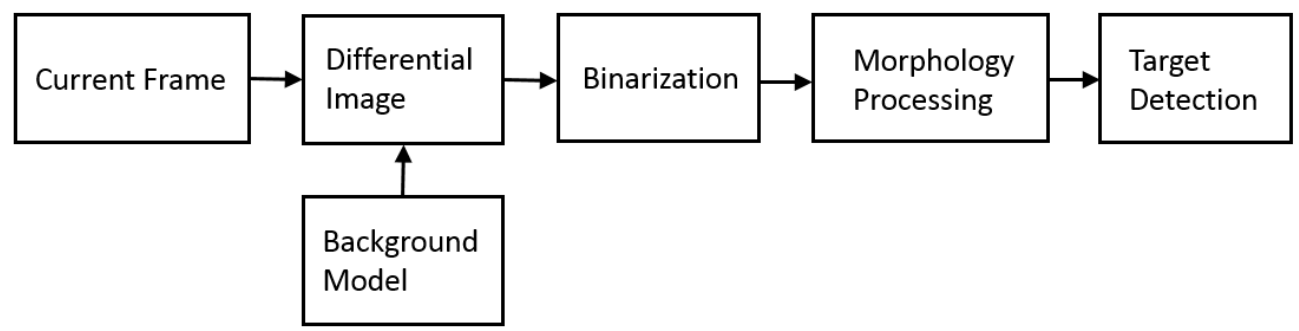

Fig. 1. Block diagram of the traditional background subtraction method. 
The first frame as a background, $b_{k}(x, y)=f_{k}(x, y), \mathrm{k}=1$.The $f_{k}(x, y)$ is the $\mathrm{k}$ th frame image for sequence images, and the $b_{k}(x, y)$ is the $\mathrm{k}$ th frame for backgroundimages. Obtain differential image after the background subtraction:

$$
D_{k}(x, y)=\left|f_{k}(x, y)-b_{k}(x, y)\right|
$$

Select the threshold $t$, the differential image $D_{k}(x, y)$ is segmented by threshold to get binary image $B D_{k}(x, y)$ :

$$
B D_{k}(x, y)=\left\{\begin{array}{c}
255, D_{k}(x, y)>t \\
0, D_{k}(x, y) \leq t
\end{array}\right.
$$

White area represents area with the moving objects.

Over the years, increasingly background modeling algorithms have been proposed.The multi-frame [8] average method is simple, but cannot establish a precise background mode, and is updated in real time is very difficult; Gaussian mixture model (GMM) [9] approach is a statistical method, which consists of modeling the distribution of the valuesobserved over time at each pixel by a weighted mixture of Gaussians, but has flaws as large amount of computations and slow updating of background model. Surendra background update algorithm can adaptively get background model to calculate the amount is relatively small, it can be real-time implementation. By using an adaptive threshold algorithm, improved Surendra background update algorithm, and this method can reduce the lighting changes, moving background model affected by environmental changes and extract reliable.

\subsection{Background updating with adaptivebinarization threshold}

Surendra background updating algorithm to estimate the difference between the brightness of the background image. If the luminance value is greater than a threshold value $(T)$, the corresponding background image pixel position unchanged. Otherwise, replace the background image is updated with the current frame.

The algorithm can be analyzed according to the following steps:

1.Set the first frame image $f_{1}(x, y)$ as background model $b_{1}(x, y)$.

2.Calculate the difference image of the current frame, and then select the threshold $\mathrm{T}$ (generally for 27) into the binary conversion:

$$
g_{k}(x, y)=\left\{\begin{array}{l}
1,\left|f_{k}(x, y)-b_{k-1}(x, y)\right|>T \\
0,\left|f_{k}(x, y)-b_{k-1}(x, y)\right| \leq T
\end{array}\right.
$$

3.In the second step $g(x, y)$ is used to mark the location of the moving target pixel, updates the background image $b_{k}(x, y)$ defined:

$$
b_{k}(x, y)=\left\{\begin{array}{c}
b_{k-1}(x, y), g_{k}(x, y)=0 \\
\alpha f_{k}(x, y)+(1-\alpha) b_{k-1}(x, y), g_{k}(x, y)=1
\end{array}\right.
$$

The $b_{k}(x, y), g_{k}(x, y)$ are the brightness for background image and the prospects image in (x, y); $f_{k}$ for input $k f$ rames.The use of $\alpha$,which is the background update coefficient, when $\alpha$ is high can quickly adapt to the scene of some of the changes, the noise is often mixed with come in, makes the difference of the result is not ideal;When $\alpha$ is lowalthough the background update rate relatively stable, but the real time performance is not satisfied.

4.Iteration $k=k+1$ return to step (2), when iteration $k=k_{\max }$, the iteration ends. At this time $b_{k}(x, y)$ can be regarded as the background model.

This background update algorithm for large and fast moving target detection effect is poorer, produces the "double", make the detection of moving targets is larger than the original motion target; When objects move from static to leave background, may miss the background show area by moving target detection, and will not be updated.Three frame difference method to detect the target contour is closer to the original target, but for the target overlap between the three frame is still not easy to detect, there is still a large hollow phenomenon.Aiming at the shortcomings of the above background updating method, to improve the method of step 2, four frame difference method used here, using this method can accurately detect the target contour of the region.

Set $f_{k}(x, y), f_{k+1}(x, y), f_{k+2}(x, y), f_{k+3}(x, y)$ as adjacent four frames image in image sequences. The difference image processed by the four frame difference methodformula is as follows: 


$$
\begin{gathered}
d_{n k}=\left\{\begin{array}{c}
1,\left|f_{k+2(n-1)}(x, y)-f_{k+1+2(n-1)}(x, y)\right|>R+\Delta r_{n} \\
0,\left|f_{k 2(n-1)}(x, y)-f_{k+1+2(n-1)}(x, y)\right| \leq R+\Delta r_{n}
\end{array}\right. \\
\Delta r_{n}=\varepsilon \frac{1}{M \times N} \sum_{x=0}^{M-1} \sum_{y-0}^{N-1}\left|f_{k+2(n-1)}(x, y)-f_{k+1+2(n-1)}(x, y)\right| \\
d_{k}=d_{1 k} \cap d_{2 k} \\
g_{k}(x, y)=\left\{\begin{array}{c}
1, d_{k}=1 \\
0, d_{k}=0
\end{array}\right.
\end{gathered}
$$

In formula (5) and (6), $\mathrm{n}$ is the number of difference image, which can be set 1 or 2 . Ris fixed threshold. $\Delta r_{1}$ and $\Delta r_{2}$ are dynamic threshold, which reaction to the video sequence in lighting conditions, when the light changes obviously dynamic threshold increases. So that the effects of the light changes can be effectively suppressed.And when the image illumination change is small, the dynamic threshold is a small value.eis inhibition coefficient,which reference value is $2 . M \times N$ for each size of the image, and its numerical value equal to the number of pixels in the detection area.

\subsection{Adaptive background updating strategies}

After four differential detection of moving objects visible area, set $b m(x, y)$ as moving objects area by the fourdifferential detection, $b v(x, y)$ as the visible area for background detection. Then the detection rules are as follows:

1. If $b m(x, y) \notin\left|f_{k+1}-f_{k}\right| \geq R+\Delta r_{1} \& \& b v(x, y) \notin\left|f_{k+3}-f_{k+2}\right| \cdot\left|f_{k+1}-f_{k}\right| \geq R+\left(\frac{\Delta r_{1}+\Delta r_{2}}{2}\right)$

Then $\operatorname{bm}(x, y)$ is the re-exposedbackground region.

2. If $b v(x, y) \in\left|f_{k+1}-f_{k}\right| \geq R+\Delta r_{1} \& \& b v(x, y) \notin\left|f_{k+3}-f_{k+2}\right| \cdot\left|f_{k+1}-f_{k}\right| \geq R+\left(\frac{\Delta r_{1}+\Delta r_{2}}{2}\right)$

Then $b v(x, y)$ is the visible background area.

Considering that the $\operatorname{bm}(x, y)$ in the previous frame is covered by moving objects. It is then classified by updatingrules into the background. The background updating algorithm in step (3) is improved in accordance with therules, it is listed follows:

If $g_{k}(x, y)=0, b_{k}(x, y)=b_{k-1}(x, y)$.

If $g_{k}(x, y)=1$,

1. $b_{k}(x, y) \in b m(x, y)$,then update according to the following rules:

$a_{1} f_{k}(x, y)+\left(1-a_{1}\right) b_{k-1}(x, y)$

Taking into consideration that thebm $(x, y)$ point in the previous frame is covered by moving object, and is re-exposed in the current frame, so the updating rate $a_{1}$ should be greater than $a_{2}$

2. $b_{k}(x, y) \in b m(x, y)$,then update according to the following rules:

$a_{2} f_{k}(x, y)+\left(1-a_{2}\right) b_{k-1}(x, y)$

When a new frame background environment change is bigger, need larger update rate, and vice versa.According to the demand of adaptive update rate $a_{1}$ is defined as follows:

$$
\mathrm{a}_{2}=\beta\left(1-\frac{\left|\mu_{0, \mathrm{k}}-\mu_{0, \mathrm{k}-1}\right|}{\max \left|\mu_{0, \mathrm{k}}-\mu_{0, \mathrm{k}-1}\right|}\right)
$$

$\beta$ is constant in the range $[0.01,0.2], \mu_{0, k}$ and $\mu_{0, k-1}$ are respectively on the current frame and background region after $\mathrm{A}$ frame and background difference grey value.By the adoption ofthis strategy in establishing background model, with the quick movement of moving objects, more accurate backgroundmodel can be acquired when moving objects exist.

\section{Morphology Processing}

For the motion image after binarization border region is not smooth and moving targets have empty case, first the smaller structural element is adopted to image an open operation, remove the 
isolated noise, using the structural elements of the image for a larger closed operation, and fill the empty goal.After morphological processing, binarization of the noise of the motion picture and small gap has been removed, inner cavity was filled in. The connectivity analysis, can produce multiple connected regions. In addition to the real motion target area outside, still can produce small connected regions. At this point to connectivity detection area threshold $T_{1}$, comparing to all connected area, if the area is more than threshold $T_{1}$ positioning moving targets, or to eliminate it, eventually get to the real motion target area.

\section{Experimental Results}

In this experimental, the video sequence "bike.avi" is used to assess the effectiveness of the proposed algorithm in this paper. The video image as a bicycle through the streets with interference such as illumination change and leaves. We use three different methods to detect the moving object in video image sequences. The experimental results are shown below:

(a) The original video image; (b) The background obtained by basic surendra mathod; (c) The background by the method in this paper; (d) The moving object detected by two frames difference method; (f)The moving object detected by surendra method. (e) The moving object detected by the method in this paper.

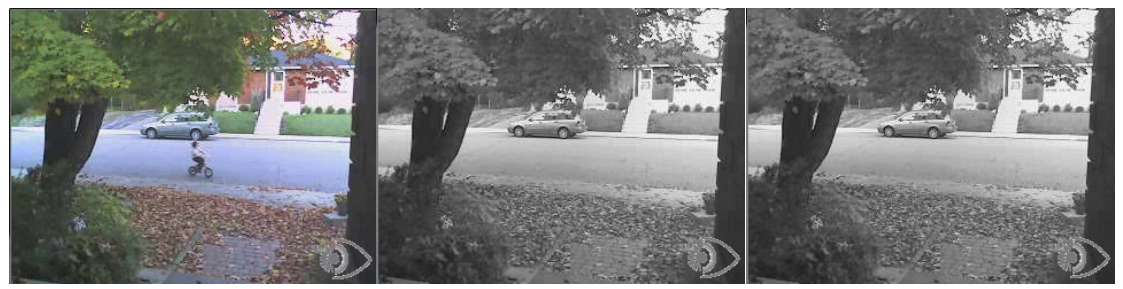

(a) Vidio frame image (b) Background model 1 (c) Background model 2

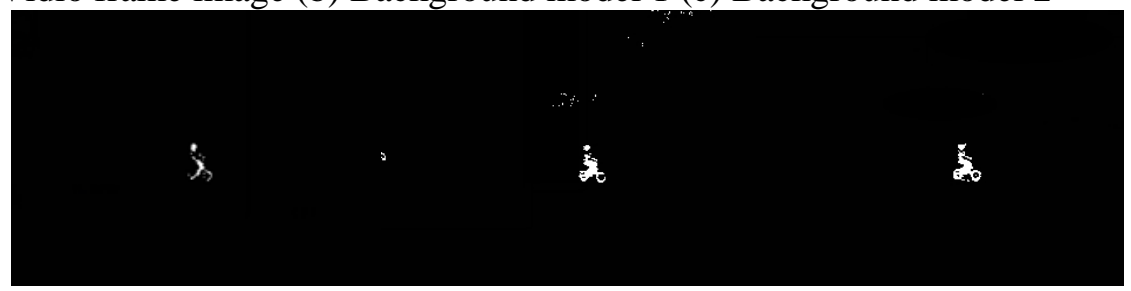

(d) Method1 (e) Method2 (f) Method3

Fig. 2.Experimental results.

Experimental results show that the two frame difference method has the obvious phenomenon of moving targets losing. And also exposed the traditional frame difference method disadvantage that moving objectappeared discontinuous "hollow" phenomenon. The movingtarget detection effectof surendra algorithm is superior to the interframe difference method, but due to the light changes, the final detection image has noise.The experimental results show that the former two algorithms are not clear and accurately extract the moving object, which makes the moving target tracking and recognition difficult.Theproposed algorithm to detect the target contour is closer to the goal itself, and solve the defects such as light interference and target contour discontinuous.

\section{Summary}

Background subtraction and frame difference method are compared between algorithms for target detection, but the use of two methods has its specific environment, combining the two methods, this paper puts forward an adaptive background updating, and combined with four method of moving object detection algorithm, frame difference and the dynamic threshold respectively into motion detection and background updating, mutations in effectively overcome the light effect and solve the problems such as double, better able to detect the moving targets. 


\section{References}

[1] Ou Yang. Moving object detecting method in video [J]. Aerospace and Electronic Systems Magazine, IEEE, Volume23, Issue 1, Jan, Page(s): 18-20(2008)

[2] Ahmed. Elgammal, Background subtraction: theory and practice, Wide AreaSurveillance, Springer, Berlin, Heidelberg (2014) 1-21.

[3] T. Bouwmans, Recent advanced statistical background modeling for foreground detection-a systematic survey, Recent Patents Comput. Sci. 4 (3)(2011) 147-176.

[4] Brutzer S, Hoferlin B, Heidemann G. Evaluation of background subtractiontechniques for video surveillance, in: IEEE Conference on Computer Vision andPattern Recognition (CVPR), 2011, pp. 1937-1944.

[5] T Bouwmans, F El Baf, B Vachon, Background modeling using mixture ofgaussians for foreground detection—a survey, Recent Patents Comput. Sci. 1(3) (2008) 219-237.

[6] Elektrik-Elektronik Mühendisligi Bölümü, Mustafa Kemal Universitesi, Antakya, Motion detection in movingpictures. Processding of the 13th Signal Processing and Communication Applications Conference,6(132)187-190(2005)

[7] Duéñez-Guzmán, Edgar A.; Rojas, Raúl; Cuevas, Erik. Unsupervised measures for parameter selection forbinarization algorithms. Pattern Recognition, 44(3) 491-502(2011)

[8] Xiaofeng Li, Zhonghui Mei. Algorithm of background extraction based on statistics of histogram combining withmulti-frame average. Nanjing Journal of Nanjing University of Posts and Telecommunications(Natural Science).28(6), 74-77(2008)

[9] Li Li, Jininq Xu. Moving human detection algorithm based on Gaussian mixture model[J]. 29th Chinese ControlConference. 10(156) 2853-2856(2010) 